\title{
Venöz tromboembolizmde tanı yöntemleri
}

\author{
Diagnostic methods in venous thromboembolism
}

\author{
Şahin Çepni, Osman Tecimel
}

Ankara Şehir Hastanesi, Ortopedi ve Travmatoloji Kliniği, Ankara

\begin{abstract}
Venöz tromboemboli, klinik olarak belirti vermeyen derin ven trombozundan, ölümcül pulmoner emboliye kadar değişen durumları içeren ve çok sık görülen bir hastalıktır. Bu potansiyel olarak öldürücü komplikasyon, en sık majör travma veya ortopedik cerrahiye ikincil olarak gözlenir. Pulmoner venöz tromboembolide tanı yaklaşımlarının ana hedefi, non-invaziv yöntemlerin kombine kullanılarak pulmoner anjiyografi gereksiniminin azaltılmasıdır. Mortaliteyi azaltmak için, venöz tromboemboli ve ölümcül komplikasyonu olan pulmoner emboli tanılarının hızlı bir şekilde konulması gerekmektedir.
\end{abstract}

Anahtar sözcükler: derin ven trombozu; pulmoner emboli; tromboemboli

\begin{abstract}
Venous thromboembolism is a very frequent condition that comprises a wide range of pathologies including the clinically subtle deep vein thrombosis and the potentially lethal pulmonary embolism. This potentially fatal complication is frequently seen during major trauma or secondary to orthopedic surgery. The diagnostic approach to pulmonary venous thromboembolism aims at successfully combining non-invasive approaches while reducing the need for pulmonary angiography. Venous thromboembolism and its fatal component, pulmonary embolism, should be diagnosed and treated urgently in order to decrease mortality.
\end{abstract}

Key words: deep vein thrombosis; pulmonary embolism; thromboembolism
$\mathbf{V}$ enöz tromboembolizmin (VTE) nedeni, çoğunlukla bacak derin venlerinde oluşan trombüslerdir. Bu trombüslerden bir kısmı koparak dolaşım yolu ile akciğerlere gelir ve pulmoner tromboembolizme (PTE) yol açar. Hastanede yatmakta olan hastaların tümünde en az bir, yaklaşık \%40'ında ise, üç ya da daha fazla sayıda venöz tromboembolizm riski bulunur. Bu nedenle VTE, önlem alınmazsa, hastane morbidite ve mortalitesinin en önemli nedenidir. ${ }^{[1,2]}$ VTE'nin en önemli komplikasyonu pulmoner emboli (PE) olup, PE'li olguların yaklaşık \%10'u ölümle sonuçlanmaktadır. ${ }^{[3]}$

Klinik bulgular ve semptomlar VTE tanısını koymada oldukça yetersiz kalmakta, tanı koydurucu objektif testlere ihtiyaç duyulmaktadır. ${ }^{[4]}$ Ancak, testleri uygulamadan önce klinik değerlendirme ile risk sınıflaması, doğru tanı için önemli ipuçları verir. Günümüzde test öncesi ön klinik değerlendirme, gerek radyolojik ve gerekse biyokimyasal testlere desteklenerek daha kolay, daha ucuz ve doğru tanı koyma rehberleri/algoritmaları geliştirilmiştir.
Mevcut tanı yöntemleri, girişimsel olan (invaziv), girişimsel olmayan (non-invaziv), ve biyokimyasal yöntemler olarak gruplara ayrılabilir. Girişimsel olan ve olmayan tanı yöntemleri, ven içindeki pihtının görüntülenmesi ve ven içi kan akımının değişmelerinin gösterilebildiği yöntemler olarak tanımlanmaktadır.

\section{KLINIK DEĞERLENDIRME}

Klinik olarak, sessiz VTE'nin hangi oranda tanınabildiği belirsizdir. ${ }^{[4,5]}$ Klinik olarak belirti ve bulgu verenlerin bir kısmına da tanı konulamamaktadır. ${ }^{[6]}$ Semptomatik derin ven trombozu (DVT) olan olgularda, etkilenen uzuvda ağrı, şişlik, ven trasesi boyunca hassasiyet, eritem veya siyanoz görülebilmektedir. Bu bulgular DVT'ye özgü olmayıp, diğer hastalıklarda da görülebilir. DVT tanısına alternatif olası tanıları; sellülit, yüzeyel flebitler, yırtılmış Baker kisti, diz eklemi problemi, kas absesi, kas içi hematom, diğer kasiskelet problemleri ve post-flebitik sendrom olarak sayabiliriz. ${ }^{[7,8]}$

- İletişim adresi: Doç. Dr. Osman Tecimel, Ankara Şehir Hastanesi, Üniversiteler Mahallesi Bilkent Caddesi No:1, Çankaya, Ankara Tel: 0505 - 4002670 e-posta: drtecimel@gmail.com

- Geliş tarihi: 8 Mayıs $2019 \quad$ Kabul tarihi: 6 Ağustos 2019 
Wells skorlaması (WS), olasılık gruplarının kolay saptanması ve standardize olması nedeniyle günümüzde sıklıkla kullanılmaktadır (Tablo 1). ${ }^{[8]}$ WS sisteminin bir diğer avantajı da, DVT dışındaki alternatif tanı olasılıklarını da puanlama yönünden dikkate almasıdır.

Sık kullanılan hemoglobin, hematokrit, platelet, beyaz küre, periferik yayma gibi kan tetkikleri ile elektrokardiyografi (EKG), akciğer grafisi, arter kan gazları (AKG) gibi laboratuvar yöntemleri, daha çok ayırıcı tanıda yardımcı olur. EKG anormallikleri sıktır, ancak özgül değildir. PTE sağ kalpte zorlanma yaratabilecek boyutta olduğu zaman, buna ait anormallikler -aks değişikliği, taşikardi, aritmiler, SI, QIII ve TIII paternisaptanabilir. AKG'lerde, pulmoner vasküler yataktaki kesintiyle orantılı sağdan-sola şantın neden olduğu hipoksemi, alveoler-arteriyel $\mathrm{PO}_{2}$ gradientinde artış ve alveoler hiperventilasyona bağlı hipokapni gözlenebilir. Her iki testin normal olması tanıyı ekarte ettirmez. Akciğer grafisinde, genellikle normal ve/veya sıklıkla özgül olmayan anormallikler vardır. Sık bulunabilen anormallikler; diyafragmada yükselme, lineer atelektaziler, az miktarda plevral effüzyon ve fokal infiltrasyondur. Tepesi hilusa bakan üçgen şeklindeki opasite, pulmoner arterlerde kesinti, hipovasküler alan nadir ve/veya kontrol akciğer grafisi olmadan saptanabilmesi güçtür.

\section{BIYOKIMYASAL DEĞERLENDIRME D-Dimer}

Koagülasyon sonu fibrin yıkım ürünlerinden D-dimerin kandaki seviyesinin VTE tanısındaki değerini araştıran birçok yayın bulunmaktadır. ${ }^{[10,11]}$ Bu test, tanıyı desteklemekten çok, klinik olasılık düşük ve risk faktörü bulunmayanlarda ekarte etme yönünden daha değerli gibi görünmektedir. Klinik olarak orta ve düşük

Tablo 1. Wells skorlaması (WS)

\begin{tabular}{lc}
\hline Pulmoner emboli olasılığı: Wells kriterleri & Skor \\
\hline DVT klinik bulguları & 3 \\
PE dışı tanı olasılığının düşük olması & 3 \\
Kalp hızı >100/dk & 1,5 \\
$\begin{array}{l}\text { Immobilizasyon ( } \geq 3 \text { gün) veya son dört gün içerisinde } \\
\text { cerrahi girişim }\end{array}$ & 1,5 \\
Öyküde DVT/PE & 1,5 \\
Hemoptizi & 1 \\
Malignite & 1 \\
\hline WS >4 ise PE olası; tanısal testler yapılmalı. & \\
Kaynak: Cutts BA, et al., Am J Obstet Gynecol 2012. ${ }^{\left[{ }^{9]}\right.}$ &
\end{tabular}

olasılık grubuna giren hastalarda, 0,5 $\mu g^{\prime} ı$ altındaki değerler pratik olarak VTE tanısını ekarte eder.[12] Cerrahi girişim, böbrek hastalıkları, travma, maligniteler, ağır enfeksiyonlar, sistemik lupus eritematozus, gebelik vb. durumlarda da test pozitif bulunabilir. ${ }^{[10]}$

\section{Kardiyak Troponin}

Troponin I ve troponin T, kalp kasının kontraksiyonunda fonksiyon görür; miyokard hasarında kana geçer. PTE'ye bağlı sağ ventrikül disfonksiyonunda hatta prognozun belirlenmesinde troponin seviyelerinin kanda yükselmesinin anlamlı olabileceğini gösteren çalışmalar bulunmaktadır. ${ }^{[13,14]}$

\section{Kontrastlı Venografi}

DVT tanısında hala altın standart olarak kabul edilmektedir. ${ }^{[15]}$ Ancak, son yıllarda girişimsel olmayan testlerin sayısının artması ve bu testlerin kolay uygulanabilirliği ve güvenilirliği nedeniyle pek kullanılmamaktadır. Venografınin girişimsel, ağrılı, teknik olarak zor olması, donanıma ihtiyaç duyulması ve değerlendirme için ise tecrübeli eleman gerekliliği gibi bazı dezavantajları vardır. Son yıllarda, klinik olarak olasılık saptanmasının girişimsel olmayan testlerle birleştirilmesi ve hem güvenilir hem de emniyetli sonuçlar alınması, venografi ihtiyacını azaltmıştır. ${ }^{[16,17]}$ Venografi, asemptomatik olan olgularda kesinlikle tarama testi olarak yapılmamaktadır.

\section{İmpedans Pletismografi (IPG)}

Alt ekstremitelerdeki hacim değişikliklerini saptayan, girişimsel olmayan bir yöntemdir. Bu testlerin doğru sonuç vermesi için, hastanın muayene edilen damarında daha önceden tromboz geçirmemiş olması ve bu damara dıştan bası olmaması gerekir.

\section{Venöz Kompresyon Ultrasonografisi (US)}

DVT tanısında çeşitli US tipleri (konvansiyonel, dupleks ve renkli Doppler US) kullanılabilmektedir. Proksimal-popliteal venlerin hafif bir basınçla komprese edilip edilememesi, tanıda yardımcı olmakta ve bu yönteme venöz kompresyon ultrasonografisi (VKUS) denilmektedir. Popliteal veya daha üst seviyedeki venlerde komprese edilemeyen bir segmentin varlığı, proksimal DVT tanısı koydurur. ${ }^{[7]}$ Girişimsel olmayan bir yöntem olması, her yerde yapılabilmesi, ucuz olması ve tekrarlanabilmesi gibi avantajları vardır. Kompresyon US'nin proksimal DVT tanısındaki duyarlılığı \%95, özgüllüğü ise \%96'dır. ${ }^{[7]}$ Ancak, izole baldır ven trombozunda bu oranlar düşmekte, sırası ile $\% 60$ ve $\% 70$ olmaktadır. ${ }^{[7]}$ En önemli dezavantajı, izole distal tromboz tanısında duyarlılık ve özgüllüğün az olmasıdır. 


\section{Bilgisayarlı Tomografi (BT)}

Batın ve pelvis içi büyük venlerdeki trombüsü saptamada konvansiyonel flebografiden daha yararlıdır. Lümen içi trombüsün saptanması, yeni trombüsün eskisinden ayırt edilebilmesi ve damarlara dıştan basıların gösterilebilmesi gibi yararlı yanları vardır. ${ }^{[4,18]}$

\section{Ventilasyon/Perfüzyon (V/Q) İzotop Sintigrafisi}

Pulmoner embolinin tanısında ventilasyon/perfüzyon sintigrafisinin duyarlılığı yüksek ancak özgüllüğü düşüktür. ${ }^{[19]}$ Pnömoni, atelektazi, tümöral lezyonlar, kronik obstrüktif akciğer hastalıkları, akciğer ödemi gibi patolojiler de perfüzyon bozukluklarına neden olabilir. ${ }^{[20]}$ Sintigrafik olarak PE'nin karakteristik bulgusu; ventilasyon normal iken, perfüzyonda defekt olmasıdır (mismatched defect).

\section{Manyetik Rezonans (MR) Görüntüleme}

MR görüntüleme, pahalı ve etkinliği gösterilmemiş bir yöntemdir. Spiral BT'de olduğu gibi, segmenter arterlerin periferindeki tıkanmalarda duyarlılığı giderek azalır. Özellikle inferior vena kava ve iliak venlerin trombozlarının tanısında US'den üstündür. ${ }^{[21]}$

\section{Pulmoner Kateter Anjiyografi (PKA)}

PTE tanısında altın standart olarak tanımlanan PKA, son yıllarda yerini daha az invaziv, uygulanabilme olasılığı çok daha yüksek ve kolay olan helikal BT'ye terketmektedir. ${ }^{[22,23]}$ PKA'nın tanısal etkinliği, izole ve subsegmental emboliler için düşüktür. ${ }^{[24]}$ PKA, günümüzde tanıdan çok tedavi amaçlı kullanılmakta olup, bu amaçla kateter eşliğinde tromboliz, perkütan embolektomi, trombektomi ve pıhtı aspirasyonu işlemleri yapılabilmektedir. ${ }^{[25]}$

\section{Bilgisayarlı Tomografi Anjiyografi (BTA)}

Pulmoner BTA, günümüzde pek çok merkezde $\mathrm{PE}$ şüphesi ile başvuran olgularda en sık kullanılan görüntüleme yöntemi haline gelmiştir. ${ }^{[26,27]}$ PE tanısında en önemli tanısal görüntüleme yöntemi olarak kabul görmektedir. ${ }^{[28]}$ Yapılan çalışmalarda, PE şüphesi ile görüntüleme yapılan hastaların ancak \%8-20'lik bölümünde emboli saptanırken, geriye kalan hastaların büyük kısmında altta yatan diğer patolojiler görülmüştür. ${ }^{[26,29]}$ Ancak, iyonizan radyasyona ve nefrotoksik kontrast maddelere maruziyet BT'nin temel dezavantajlarıdır. Çeşitli çalışmalarda pulmoner BTA'nın özgüllük ve duyarlılık aralıkları, sırasıyla \%53-100 ve \%83-100 olarak bildirilmiştir. ${ }^{[30,31]}$

\section{Ekokardiyografi}

Ekokardiyografi PE tanısında rutin olarak kullanılmamakla birlikte, tanı konulan hastalarda riskin belirlenmesi, prognozun tayini ve şok tablosu ile acile başvuran ve masif emboliden kuşkulanılan olgularda yapılacak ilk inceleme olmalıdır. ${ }^{[32]}$ Ekokardiyografi, sağ kalp odacıklarının değerlendirilmesinin yanı sıra PE'nin ayırıcı tanısında yer alan aort disseksiyonu, perikardiyal hastalıklar, miyokard infarktüsü ve valvüler yetmezlik gibi tanıların da dışlanmasına yardımcı olabilir. Ekokardiyografi, PE'nin hemodinamik etkilerinin gösterilmesinin yanında prognozun belirlenmesinde de etkindir. Akut PE'de ekokardiyografide sağ ventrikülde art yüke bağlı disfonksiyon bulunması, artmış mortalite ve komplikasyonlar ile ilişkilidir. ${ }^{[3]}$

\section{KAYNAKLAR}

1. Geerts WH, Bergqvist D, Pineo GF, Heit JA, Samama CM, Lassen MR, Colwell CW. Prevention of Venous Thromboembolism. Chest 2008;133(6):381S-453S. Crossref

2. Goldhaber SZ, Savage DD, Garrison RJ, Castelli WP, Kannel WB, McNamara PM, Gherardi G, Feinleib M. Risk factors for pulmonary embolism. The Framingham Study. Am J Med 1983;74(6):1023-8. Crossref

3. Stein PD, Henry JW. Prevalence of acute pulmonary embolism among patients in a general hospital and at autopsy. Chest 1995;108(4):978-81. Crossref

4. Lensing AWA, Hirsh J, Ginsberg JS, Büller HR. Diagnosis of venous thrombosis. In: Colman RW, Hirsh J, Marder VJ, Clowes AW, George JN, editors. Hemostasis and Thrombosis. Philadelphia, PA: Lippincott Williams \& Wilkins, Inc.; 2001. pp.1277-301.

5. Moser KM, Fedullo PF, LittleJohn JK, Crawford R. Frequent asymptomatic pulmonary embolism in patients with deep vein thrombosis. JAMA 1994;271(3):223-5. Crossref

6. Goldhaber SZ, Hennekens CH, Evans DA, Newton EC, Godleski JJ. Factors associated with correct antemortem diagnosis of major pulmonary embolism. Am J Med 1982;73(6):822-6. Crossref

7. Bauer KA. Management of inherited thrombophilia. UpToDate version 10.2, April 26, 2002.

8. Wells PS, Anderson DR, Bormanis J, Guy F, Mitchell M, Gray L, Clement C, Robinson KS, Lewandowski B. Value of assessment of pretest probability od deep-vein thrombosis in clinical management. Lancet 1997;350(9094):1795-8. Crossref

9. Cutts BA, Dasgupta D, Hunt BJ. New directions in the diagnosis and treatment of pulmonary embolism in pregnancy. Am J Obstet Gynecol 2013;208(2):102-8. Crossref

10. Goldhaber SZ, Simons GR, Elliot CG, Haire WD, Toltzis R, Blacklow SC, Doolittle MH, Weinberg DS. Quantitative plasma D-dimer levels among patients undergoing pulmonary angiography for suspected pulmonary embolism. JAMA 1993;270(23):2819-22. Crossref

11. Oger E, Lebroyer C, Bressollette L, Nonent M, Le Moigne E, Bizais Y, Amiral J, Grimaux M, Clavier J, Ill P, Abgrall $J-F$, Mottier D. Evaluation of a new, rapid and quantitative D-dimer test in patients with suspected pulmonary embolism. Am J Respir Crit Care Med 1998;158(1):65-70. Crossref

12. Bates SM, Grand'Maison A, Johnston M, Naguit I, Kovacs MJ, Ginsberg JS. A latex D-Dimer reliably excludes venous thromboembolism. Arch Intern Med 2001;161(3):447-53. Crossref 
13. Meyer $T$, Binder L, Hruska $N$, Luthe $H$, Buchwald $A B$. Cardiac troponin I elevation in acute pulmonary embolism is associated with right ventricular dysfunction. J Am Coll Cardiol 2000;36(5):1632-6. Crossref

14. Giannitis E, Müller-Bardorff M, Kurowski V, Weidtmann B, Wiegand U, Kampmann M, Katus HA. Independent prognostic value of cardiac troponin $\mathrm{T}$ in patients with cofirmed pulmonary embolism. Circulation 2000;102(2):211-7. Crossref

15. Lensing AWA, Büller HR, Prandoni P, Batchelor D, Molenaar AHM, Cogo A, Vigo M, Huisman PM, ten Cate JW. Contrast venography, the gold Standard for the diagnosis of DVT. improvement in observer agreement. Thromb Haemost 1992;67(1):8-12. Crossref

16. Lensing AWA, Prandoni $P$, Prins $M H$, Büller HR. Deep-vein thrombosis. Lancet 1999;353(9151):479-485. Crossref

17. Tapson VF, Carroll BA, Davidson BL, Elliott CG, Fedullo PF, Hales CA, Hull RD, Hyers TM, Leeper KV Jr, Morris TA, Moser KM, Raskob GE, Shure D, Sostman HD, Taylor Thompson B. The Diagnostic approach to acute venous thromboembolism. Clinical Practice Guideline. Am J Respir Crit Care Med 1999;160(3):1043-66. Crossref

18. Lee AYY, Levine MN. Cancer and Thrombosis. Connecticut: Chase Medical Communications, Inc.; 2002.

19. Reinartz P, Wildberger JE, Schaefer W, Nowak B, Mahnken $\mathrm{AH}$, Buell $\mathrm{U}$. Tomographic imaging in the diagnosis of pulmonary embolism: a comparison between $\mathrm{V} / \mathrm{Q}$ lung scintigraphy in SPECT technique and multislice spiral CT. J Nucl Med 2004;45(9):1501-8.

20. Bajc M, Olsson B, Palmer J, Jonson B. Ventilation/perfusion SPECT for diagnostics of pulmonary embolism in clinical practise. J Intern Med 2008;264(4):379-87. Crossref

21. Spritzer CE, NorconkJJ, Sostman HD, Coleman RE. Detection of deep venous thrombosis by magnetic resonance imaging. Chest 1993;104(1):54-60. Crossref

22. Pond GD, Ovitt TW, Capp MP. Comparison of conventional pulmonary angiography with intravenous digital subtraction angiography for pulmonary embolic disease. Radiology 1983;147(2):345-50. Crossref

23. Musset D, Rosso J, Petiptretz P, Girard P, Hauuy MP, Simonneau G, Labrune M, Duroux P. Acute pulmonary embolism: Diagnostic value of digital substruction angiography. Radiology 1988;166(2):455-9. Crossref

24. Stein PD, HenryJW, GottschalkA. Reassessment of pulmonary angiography for the diagnosis of pulmonary embolism: relation of interpreter agreement to the order of the involved pulmonary arterial branch. Radiology 1999;210(3):689-91. Crossref
25. Tajima H, Murata S, Kumazaki T, Nakazawa K, Ichikawa K, Yamamoto T, Tanaka K, Takano T. Recent advances in interventional radiology for acute massive pulmonary thromboembolism. J Nippon Med Sch 2005;72(2):74-84. Crossref

26. Tresoldi S, Kim YH, Baker SP, Kandarpa K. MDCT of 220 consecutive patients with suspected acute pulmonary embolism: incidence of pulmonary embolism and of other acute or non-acute thoracic findings. Radiol Med 2008;113(3):373-84. Crossref

27. Ritchie G, McGurk S, McCreath C, Graham C, Murchison JT. Prospective evaluation of unsuspected pulmonary embolism on contrast enhanced multidetector CT (MDCT) scanning. Thorax 2007;62(6):536-40. Crossref

28. Remy-Jardin M, Pistolesi M, Goodman LR, Gefter WB, Gottschalk A, Mayo JR, Sostman HD. Management of suspected acute pulmonary embolism in the era of CT angiography: a statement from the Fleischner Society. Radiology 2007;245(2):315-29. Crossref

29. Lee EY, Kritsaneepaiboon S, Zurakowski D, Arellano CM, Strauss KJ, Boiselle PM. Beyond the pulmonary arteries: alternative diagnoses in children with MDCT pulmonary angiography negative for pulmonary embolism. AJR Am J Roentgenol 2009;193(3):888-94. Crossref

30. Stein PD, Fowler SE, Goodman LR, Gottschalk A, Hales CA, Hull RD, Leeper KV Jr, Popovich J Jr, Quinn DA, Sos TA, Sostman HD, Tapson VF, Wakefield TW, Weg JG, Woodard PK; PIOPED II Investigators. Multidetector computed tomography for acute pulmonary embolism. N Engl J Med 2006;354(22):2317-27. Crossref

31. Rathbun SW, Raskob GE, Whitsett TL. Sensitivity and specificity of helical computed tomography in the diagnosis of pulmonary embolism: a systematic review. Ann Intern Med 2000;132(3):227-32. Crossref

32. Goldhaber SZ. Echocardiography in the Management of Pulmonary Embolism. Ann Intern Med 2002;136(9):691700. Crossref

33. Kasper W, Konstantinides S, Geibel A, Tiede N, Krause T, Just $\mathrm{H}$. Prognostic significance of right ventricular afterload stress detected by echocardiography in patients with clinically suspected pulmonary embolism. Heart 1997;77(4):346-9. Crossref 